\title{
Exploration of Ring Oscillator Based Temperature Sensors Network Accuracy on FPGA
}

\author{
Golnaz Korkian ${ }^{1}$, Navid Rahmanikia ${ }^{2}$, Hamid Noori $^{2}$, Juan Antonio Clemente ${ }^{1}$ \\ ${ }^{1}$ Computer Architecture Department, Universidad Complutense de Madrid, Madrid, Spain \\ ${ }^{2}$ Faculty of Engineering, Ferdowsi University of Mashhad, Mashhad, Iran \\ gkorkian@ucm.es,navid.rahmanikia@mail.um.ac.ir,hnoori@um.ac.ir,ja.clemente@fdi.ucm.es
}

\begin{abstract}
During the last decades, technology scaling in reconfigurable logic devices enabled implementing complicated designs which results in higher power density and on-chip temperature. Since higher operating temperature of chips is a critical problem in electronics devices, thermal management techniques are highly required. To provide a thermal map of reconfigurable logic devices, a network of sensors is needed. In this work, a ring-oscillator-based temperature sensor is used to create a sensor network. Then, a design space exploration is done among several sensor networks with the various sensor configurations including different ring oscillator length, the number of sensors in the examined network and various sampling time. We propose three criteria for exploring and comparing the efficiency of sensors network based on the thermal overhead and also measurement accuracy and precision among plenty of configurations on the Virtex-6 FPGA.
\end{abstract}

Keywords - Sensor network, Ring oscillator, Accuracy, Precision, Sampling time, Thermal overhead, FPGA.

\section{INTRODUCTION}

In the recent years, as transistors are shrinking in the reconfigurable embedded devices, there has been a noticeable increase in power consumption and especially in operating temperature. Since power consumption and temperature can interact with each other, the designers have to minimize the power consumption, the on-chip rising temperature and improve the performance and reliability of the system during the entire design flow [1].

Accurate thermal measurement techniques can improve the performance of chips particularly for multi-core systemon-chips (SoCs) [2]. To address these issues, the researchers must have a precise thermal map of chips to estimate and predict the die temperature in order to apply dynamic thermal management (DTM) techniques to maintain the operation of systems safe and reliable. In some modern field programmable gate arrays (FPGAs), i.e. Xilinx Virtex-5 and Virtex-6, there is one built-in thermal diode that gets the die temperature only in a fixed location (usually at the center of the die) [3], [4]. In the last years, the designers have paid a lot of attention to soft sensors. They have proposed different designs for soft sensors [5-17]. Some researchers utilize an array of temperature sensors based on a ring oscillator (RO). These sensors are reconfigurable and can be inserted or removed at run-time [5]. Also one of the most difficult challenges in the case of on-chip measurement is errors related to wire crosstalk and thermal noise. Hence, the accuracy and precision in soft sensors should be taken into account [11]. Design space exploration (DSE) can assist the designer to find an optimal configuration of the sensor network. In this paper, several platforms are implemented that differ in RO length and sampling time. We examine and evaluate the thermal overhead, the accuracy and precision. This work investigates the influences of different parameters on the examined sensors network.

The rest of the paper is organized as follows. Section 2 gives an overview on the related work. Section 3 presents the architecture of the measurement system. In section 4, basic parameters for evaluating the sensors network are illustrated. Experimental Results and analysis are discussed in section 5. Finally, in Section 6, the paper is concluded.

\section{RELATED WORK}

This section demonstrates the related work in temperature sensor designs, sensor calibration, array of thermal sensors and heat-generating techniques.

\section{A. Temperature Sensor Designs}

A thermal sensor based on an RO is constructed conventionally using an odd number of inverters in a loop and an AND gate for avoiding of counter overflow and reducing the self-heating effects. Also there is a counter that captures the oscillations of the RO at a fixed interval time, called as sample time or sample period, as depicted in Fig. 1.

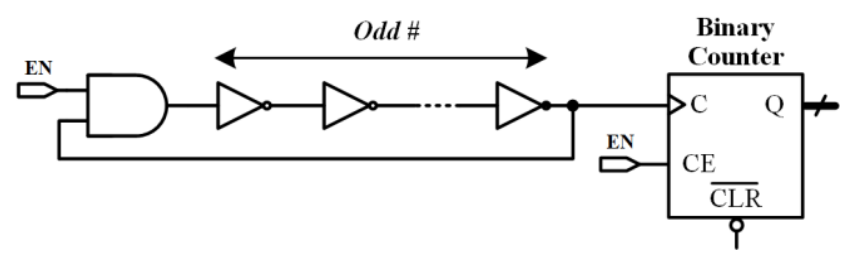

Fig.1. Conventional RO-based temperature sensor [15].

The number of inverters and interconnections delay defines the oscillation frequency as in (1):

$f=\frac{1}{t_{p}}=\frac{1}{n \times t_{D}}$

where $t_{p}$ is the oscillation period, $t_{D}$ is the propagation delay of one inverter, and $n$ is the number of inverters in the loop. 
Note that, the researchers usually use different types of counters, i.e., binary counter and residue number system (RNS) ring counter. The RNS ring counter is utilized by [7, 15], in order to make the sensor more compact. Also, the researchers design various sensors using different RO configurations. For instance, Payá-Vayá et al. propose a novel temperature sensor, which uses only 3 digital signal processor (DSP)-slices and one look-up table (LUT) on a Xilinx Virtex-6 FPGA [16]. In [11], the authors design a novel intra-chip physical sensor that relies on the setup and hold time conditions of a flip-flop on the Altera Cyclone $\Pi$. The researchers in [15] introduce a fully digital temperature sensor that occupies $37.5 \%$ fewer resources compared to other compact design (i.e. [7]) and obtains 2.72 times more sensitivity.

\section{B. Sensor Calibration}

One of the most popular approaches for measuring the die temperature is to construct a temperature-dependent circuit, i.e., a ring oscillator and calibrate its output. Due to the fact that the oscillation frequency of an RO is temperaturedepend, the system needs to calibrate the sensor to convert the measured frequencies to the corresponding temperature. Different calibration techniques are presented in the literature. Some designs are based on the built-in thermal diode [3], [4] which use the system monitor in Xilinx FPGAs and intellectual property (IP) cores in Altera FPGAs [18]. Among other alternative methods, some designers utilize external devices such as temperature probe, oven, and chamber which are applied as a reference for calibration [7], [9].

\section{Sensors Network}

For measuring and preparing an accurate thermal distribution on FPGA-based systems, an array of temperature sensors based on the RO is proposed by [1-5]. In [10], the authors design an array of $4 \times 8$ sensors to demonstrate the temperature gradients and finding hotspots. Zick and Hayes present a network with over 100 sensors for measuring variations in some parameters, such as delay and IR drop caused by switching activity [7]. A thermal sensing network that has been composed of $6 \times 8$ arrays of sensors is proposed by [19] to capture the on-chip thermal map using fuzzy clustering map.

\section{Heat-Generating Methods}

One of the most important challenges to measuring the on-chip temperature is the restriction on the temperature ranges. Agne et al. [20] introduce seven ways to make the high-tech FPGAs heat using available internal resources. In this paper, we use the heat generator circuit that has been designed in [20], i.e., the one-level LUT-based heater.

\section{THE ARCHITECTURE OF THE MEASUREMENT SYSTEM}

The basic architecture of our sensors network platform is shown in Fig. 2. The system setup of all experiments consists of the main parts [15].

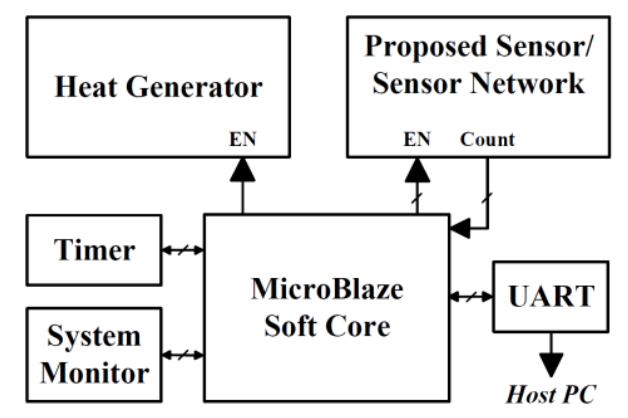

Fig. 2. Block diagram of the system architecture.

\section{A. MicroBlaze Core}

The MicroBlaze soft-microprocessor core that controls and manages all components and also run the software written in $\mathrm{C}$ for reading the sensors' data and activates/deactivates the heat generators. The MicroBlaze and all of its components operate at $100 \mathrm{MHz}$.

\section{B. Array of Temperature Sensors}

The array of temperature sensors is utilized in order to measure the temperature on different parts of the chip. The sensors are constructed using the binary counter and the RO. The construction of the RO includes a NAND gate and an even number of inverters as depicted in Fig. 3. Note that, the width of the binary counter is 15 bits.

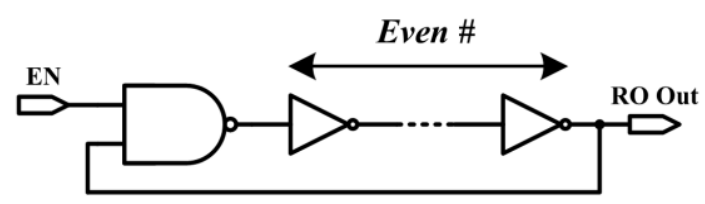

Fig. 3. Ring oscillator with an even number of inverters and a NAND gate.

\section{Heat Generator Circuits}

The heat generators are used to increase the on-chip temperature from $30^{\circ} \mathrm{C}$ to $60^{\circ} \mathrm{C}$. The processor local bus (PLB) connects the heater circuit to the MicroBlaze. The heat generator circuit consists of 10,000 one-level LUTbased oscillator which enables the maximum toggling frequency [20]. Fig. 4 shows the schematic of the one-level LUT-based heater.

\section{Timer}

The timer IP core is instantiated in order to set a fixed sampling rate, allowing counting the number of clock cycles of the RO. In this work, we explore different sampling time from $40 \mu \mathrm{s}$ to $120 \mu \mathrm{s}$.

\section{E. System Monitor}

The system monitor IP core supports the 10-bit resolution, 200 kilo-samples per second (KSPS) analog-to-digital converter (ADC) [4]. It is used to measure the temperature as well as core voltage values of the Xilinx Virtex-6 FPGA. 


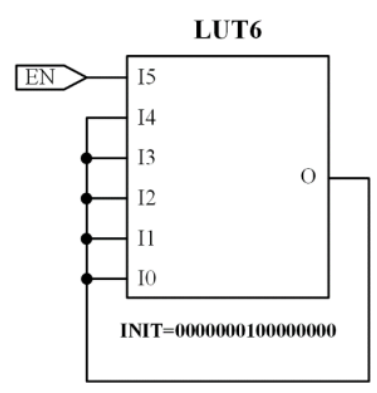

Fig. 4. Schematic of the one-level LUT-based oscillator heater.

\section{F. Universal Asynchronous Receiver/Transmitter}

In this paper, we use the universal asynchronous receiver/transmitter (UART) IP core for logging the sensors' values through RS232 to a host PC. It reports the temperature and core voltage values.

\section{BASIC PARAMETERS FOR EVALUATING THE SENSORS NETWORK}

The sensors network enables to measure the temperature of different locations of a FPGA, however, it has area, power and temperature overheads. What is clear is that there are trade-offs between the accuracy of the sensors network and overheads. In this section, we define three main metrics to evaluate different designs of sensors network. These metrics help to explore better designs in large design space.

\section{A. Thermal Overhead}

Sensors impose a thermal overhead on the design when they are activated due to the RO oscillations which vary with respect to the length of the oscillator, sampling rate and sensor configuration. As a result, calculating the thermal overhead is one of the influential factors that the designer should take into account at the design stage.

In order to measure the thermal overhead, it is necessary that all sensors are initially deactivated and then the temperature increases by activating the heat generators from $30^{\circ} \mathrm{C}$ to $60^{\circ} \mathrm{C}$. Assume it takes $t_{m}$ seconds. The temperature after $t_{m}$ seconds is called $T_{\text {withoutnet }}$. Then, the sensors and the heat generators are activated for $t_{m}$ seconds. In this case the temperature after $t_{m}$ seconds is called $T_{\text {withnet }}$ [15]. The thermal overhead $T_{O H}$ is calculated as in (2):

$T_{O H}=T_{\text {withnet }}-T_{\text {withoutnet }}$

\section{B. Accuracy}

The measurement accuracy of a sensor or a sensor network is one of the most frequently used characteristics. To calculate the accuracy for the sensor network, the wellknown root-mean-squared error (RMSE) is used to measure the error $E$. The RMSE is calculated as in (3):

$$
E=\sqrt{\frac{1}{n} \sum_{i=1}^{n}\left(P_{i-} A_{i}\right)^{2}}
$$

where $P_{i}$ is predicted value for data point $i . A_{i}$ is actual value for the data point $i$ and $\mathrm{n}$ is total number of data points.

\section{Precision}

The measurement precision illustrates the distribution of samples [11]. In this case, if the values are close together, then it has a high degree of precision. The measurement precision is calculated by the standard deviation in different sampling time. The standard deviation $\sigma$ is defined as in (4):

$$
\sigma=\sqrt{\frac{1}{n} \sum_{i=1}^{n}\left(P_{i}-\mu\right)^{2}}
$$

where $P i$ is values for the data point $i, \mu$ is mean of all values and $n$ is total number of data points.

\section{EXPERIMENTAL RESULTS}

In this section, we introduce the specification of the utilized FPGA and then discuss about calibration of the examined sensors network and analysis of the presented results.

\section{A. System Setup}

For experimental measurements, we used the Xilinx Virtex-6 ML605 FPGA development board. According to the design, the MicroBlaze soft-microprocessor operates at $100 \mathrm{MHz}$. In all our platforms, we partitioned the FPGA containing $160 \times 239$ slices into a regular grid of $10 \times 9$ tiles where each sensor is located at its center. The range of operating temperatures is from $\mathrm{T}_{\min }=30^{\circ} \mathrm{C}$ to $\mathrm{T}_{\max }=60^{\circ} \mathrm{C}$. The design consists of 5 heat-generators with 10,000 LUTs and 140 sensors in the network. We implemented different platforms with various RO lengths and sampling rates.

\section{B. Calibration Sensor Network}

Due to the different nondeterministic routing algorithms and process variation in modern technologies, the calibration of the sensors should be done separately [8]. For this reason, in order to map the measured frequencies of the RO to chip temperature, i.e., the frequency to temperature converter equation (FTC) can be obtained by regression analysis. Using the Xilinx FPGA Editor tool, we fixed the routing in $\mathrm{RO}$ in order to minimize the routing effects. For estimating temperature based on counter values, we use a regression based model. To determine the regression coefficients, we design and implement a sensor as close as possible to the system monitor and record the values of counters for different temperatures. According to the coefficients that obtained, we calibrate other sensors. However, this method results in a high RMSE. To solve this issue, each sensor is calibrated individually. Using this approach the RMSE value improves about $50 \%$.

The frequency of an RO is a function of temperature and supply voltage [21]. In this paper, a quadratic polynomial model is used to calibrate each sensor, according to equation (5): 


$$
\begin{gathered}
T(x, y)=c_{1} f^{2}(x, y)+c_{2} V_{D D}{ }^{2}+c_{3} f(x, y) \times V_{D D}+ \\
c_{4} f(x, y)+c_{5} V_{D D}+c_{6}
\end{gathered}
$$

where $f(x, y)$ and $T(x, y)$ are the frequency and the temperature of the sensor at location $(x, y)$, respectively. $V_{D D}$ is the core voltage, and $c_{i}$ is the calibration coefficient. The RMSE is provided for every sensor in various platforms.

\section{Evaluation Results and Analysis}

To evaluate the performance of different configurations of the sensors network, 9 different experiments were performed and each experiment was repeated 5 times. In these experiments, we explore to study the effects of the RO length and sampling period on the important metrics (thermal overhead, accuracy and precision). In all experiments, the width of the 15-bit counter and the number of sensors (140) are constant. Using the Xilinx FPGA PlanAhead tool, the floorplan of the sensors' placement with 5 heat generators and 140 sensors was designed. The layout of the examined sensor network is shown in Fig. 5.

According to equation (2), we calculate the thermal overhead for different configurations of sensors in the network in the range of $30^{\circ} \mathrm{C}$ to $60^{\circ} \mathrm{C}$. The results are shown in Table I. As expected, with the increase of the RO length, the thermal overhead is reduced. In the other words, thermal overhead increase due to higher oscillation frequency of the shorter RO. For example, 7 -stage RO in $80 \mu$ s generates heat about $27 \%$ more than 17 -stage RO with the same sampling period. It can be seen that the difference of the RO frequency between the slowest $(248.925 \mathrm{MHz})$ and the fastest frequency $(268.425 \mathrm{MHz})$ that occur in the maximum $\left(60^{\circ} \mathrm{C}\right)$ and the minimum $\left(30^{\circ} \mathrm{C}\right)$ of temperature respectively, in 7-stage RO in $40 \mu$ s reaches about $20 \mathrm{MHz}$. As a result, each sensor generates the different amount of heat [15]. It should be considered to get accurate results in thermal overhead, the mean of oscillation frequencies of ROs [15]. Fig 6 demonstrates the average oscillation frequency of 9 different designs.

To study the influences of the sampling time on the networks efficiency, we tried three different sampling rates including: $40 \mu \mathrm{s}, 80 \mu \mathrm{s}$ and $120 \mu \mathrm{s}$. We found that thermal overhead metric increases due to higher sampling time. Because in higher sampling period, the RO will be active longer and counts more that it results more thermal overhead, and also this affects to increase the length of the counter. For instance, respect to results presented in Table, it can be compared to the sensor network with 7-stage ROs in $40 \mu \mathrm{s}$, the $\mathrm{T}_{\mathrm{OH}}$ is $20.7 \%$ less than the sensor network with the same length but in $120 \mu \mathrm{s}$.

In [15], the authors implement 35 sensors in a network and in presented work the number of sensors is 140 . We increase the number of sensors by 4 times, since the size of the Virtex- 6 is about 5 times larger than the Virtex-5 FPGA that is utilized by [15]. According to Table II, there is a more temperature overhead in Virtex-5 LX50T, 65-nm process technology, compared to the Virtex-6 ML605, 40-nm



Fig 5. The floorplan of the sensor's placement (140 sensors) as shown from Xilinx PlanAhead tool.

Table I. RO-based temperature sensor designs in the network for variant RO length and sampling time in terms of the thermal overhead.

\begin{tabular}{|c|c|c|c|c|}
\hline $\begin{array}{c}\text { RO } \\
\text { Length }\end{array}$ & $\begin{array}{c}\text { Sampling } \\
\text { Time }(\mu \mathrm{s})\end{array}$ & $\mathrm{T}_{\mathrm{OH}}\left({ }^{\circ} \mathrm{C}\right)$ & Accuracy & Precision \\
\hline \multirow{3}{*}{7} & 40 & 0.112 & 4.09 & 0.787 \\
\cline { 2 - 5 } & 80 & 0.148 & 3.972 & 0.748 \\
\cline { 2 - 5 } & 120 & 0.319 & 4.098 & 0.668 \\
\hline \multirow{4}{*}{17} & 40 & 0.087 & 4.091 & 0.718 \\
\cline { 2 - 5 } & 80 & 0.108 & 3.918 & 0.643 \\
\cline { 2 - 5 } & 120 & 0.089 & 3.48 & 0.572 \\
\hline \multirow{3}{*}{31} & 40 & 0.0078 & 3.697 & 0.657 \\
\cline { 2 - 5 } & 80 & 0.079 & 3.466 & 0.588 \\
\cline { 2 - 5 } & 120 & 0.09 & 3.185 & 0.563 \\
\hline
\end{tabular}

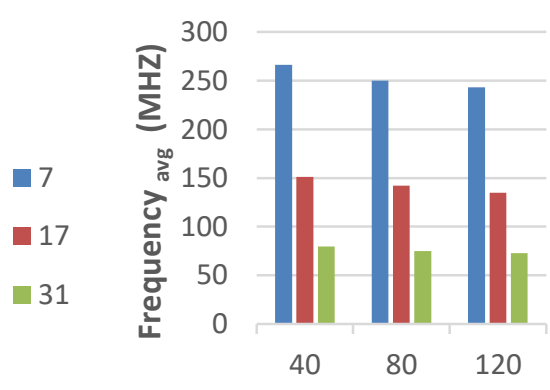

Sampling Time

Fig 6. Average oscillation frequency of 140 sensors.

process technology. For example, in the RO with 7 stages, the thermal overhead reduces about $88 \%$. Also in the experiments, the fan of the Virtex-6 FPGA cooling element 
Table II. Comparison between thermal overhead in $40 \mu \mathrm{s}$ with different stages in RO in Virtex-5 and Virtex-6.

\begin{tabular}{|c|c|c|}
\hline RO length & Virtex-5 LX50 T [15] & Virtex-6 ML605 \\
\hline 7 & 1 & 0.112 \\
\hline 17 & 0.763 & 0.087 \\
\hline 31 & 0.548 & 0.078 \\
\hline
\end{tabular}

is active. It seems that even with a $4 \mathrm{X}$ increase in the number of sensors, due to the larger area of the Virtex-6, being activated the fan and technology variation, there is less overhead in Virtex-6.

In [13] has been suggested that with increasing the number of RO's stages, the sensor sensitivity becomes higher. It has proven that for a single sensor with different stages, the precision is better for shorter ROs. We extend this concept for a network of sensors in the Virtex-6.

In order to measure the accuracy of different sensor network, according to (3), the temperature of each sensor is estimated at $30^{\circ} \mathrm{C}$ to $60^{\circ} \mathrm{C}$ and the total RMSE is reported for all 140 sensors. By calculating the average RMSEs of all sensors, the error the network can be calculated in MATLAB, as depicted in Fig 7.

To calculate the precision of the network, after sensor calibration, we obtain a standard deviation of the estimated temperatures for 140 sensors in different sampling period at a certain temperature $\left(55^{\circ} \mathrm{C}\right)$ using equation (4) that $P_{i}$ is the estimated temperature of $i$ th sensor and $n$ is 140. Fig. 8 shows the comparison results between the standard deviation of sensors network against measurement duration.

As shown in Figs. 7 and 8, the accuracy and precision improve for higher sampling periods. One of the results that can be obtained from these graphs is that for the fewer number of stages and the lower sampling rates, higher RMSE and the standard deviation can be obtained. For instance, 7-stage RO in $40 \mu$ s sampling period, we see an increase of $10 \%$ in RMSE and $16 \%$ in standard deviation compared to 31-stage RO with the same sampling period. Also, the RO with 31-stage in $120 \mu$ s has the least error and

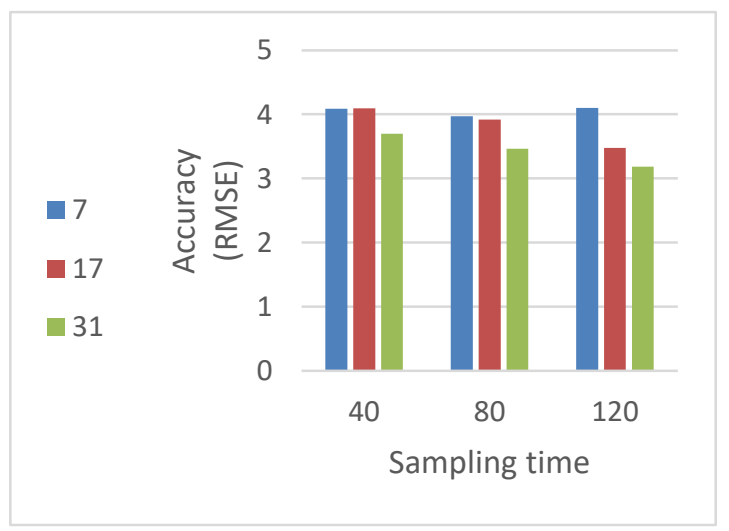

Fig 7. Accuracy of temperature measurements of sensors network.

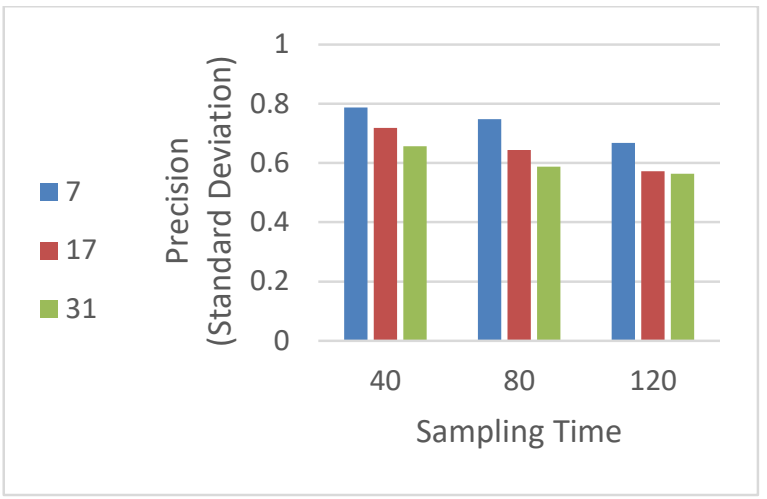

Fig 8. Precision of temperature measurements of sensors network (at $55^{\circ} \mathrm{C}$ ).

standard deviation

\section{CONCLUSION AND FUTURE WORK}

To measure the behavior of the on-chip temperature of the FPGA at runtime, a sensors network is needed. We presented an array of 140 sensors with different configurations. In comparison with [15], it has been found that due to changes in process technology, the thermal overhead has reduced about $88 \%$. One of the significant metrics in thermal overhead, accuracy and precision is the sampling period. In our experiments, the system with higher sampling rate and RO length has more precision and accuracy and also thermal overhead. However, it is seen in some sampling rates (i.e. $80 \mu \mathrm{s})$ that there is opposite results due to alternation in frequency oscillations of the RO. These experiments show that the process variation plays a more significant role than routing among state-of-the-art technologies. Although, increasing the sampling time and the counter's width, more thermal overhead and accuracy are expected.

In the future work, we intend to consider and implement the platforms with different configurations for the counters and ROs. Particularly in modern FPGAs with new technologies, the raising the sampling period (i.e. about millisecond) results the higher counter width that can have a significant effect on increasing the accuracy and thermal overhead.

\section{REFERENCES}

[1] M. Pedram, S. Nazarian, "Thermal modeling, analysis, and management in vlsi circuits, principles and methods," Proceedings of the IEEE, vol. 94, pp. 1487-1501, August 2006.

[2] S. Xie, W.T. Ng, "An all-digital self-calibrated delay-line based temperature sensor for VLSI thermal sensing and management," Integration, the VLSI Journal, vol. 51, pp. 107-117, September 2015.

[3] I. Xilinx, Virtex-5 FPGA system monitor user guide, UG192 v1.7.1 Ed., February 2011.

[4] I. Xilinx, Virtex-6 FPGA system monitor user guide, UG370 (v1.2) September 2014.

[5] S. Lopez-Buedo, J. Garrido, E. Boemo, "Dynamically inserting, operating, and eliminating thermal sensors of FPGA-based systems," IEEE Transactions on Components and Packaging Technologies, vol 25, pp. 561-566, December 2002. 
[6] M. Happe, A. Agne, C. Plessl, "Measuring and predicting temperature distributions on FPGAs at run-time," in Proceedings of the International Conference on Reconfigurable Computing and FPGAs (ReConFig), pp. 55-60, November 2011.

[7] K.M. Zick, J.P. Hayes, "Low-cost sensing with ring oscillator arrays for healthier reconfigurable systems," ACM Transactions on Reconfigurable Technology and Systems, vol 5, pp. 1-26, March 2012.

[8] P. Weber, M. Zagrabski, B. Wojciechowski, M. Nikodem, K. Kępa, K.S. Berezowski, "Calibration of RO-based temperature sensors for a toolset for measuring thermal behavior of FPGA devices," Microelectronics Journal, vol 45, pp. 1753-1763, December 2014.

[9] Y. Yue, F. Shi-Wei, G. Chun-Sheng, Y. Xin, F. Rui-Rui, “All-digital thermal distribution measurement on field programmable gate array using ring oscillators", Microelectronics Reliability, vol 55, pp. 396401, February 2015.

[10] S. Lopez-Buedo, E. Boemo, "Making visible the thermal behaviour of embedded microprocessors on FPGAs," a progress report, in Proceedings of the ACM/SIGDA International Symposium on FieldProgrammable Gate Arrays (FPGA), pp. 79-86, February 2004.

[11] G. Tarawneh, T. Mak, A. Yakovlev, "Intra-chip physical parameter sensor for FPGAS using flip-flop metastability," in Proceedings of the Field Programmable Logic and Applications (FPL), pp. 373-379, August 2012.

[12] B. Lee, K.S. Chung, B. Koo, N.W. Eum, T. Kim, "Thermal sensor allocation and placement for reconfigurable systems," ACM Transactions on Design Automation of Electronic Systems, vol 14, pp. 1-23, August 2009.

[13] J.J.L. Franco, E. Boemo, E. Castillo, L. Parrilla, "Ring oscillators as thermal sensors in FPGAs: experiments in low voltage," in
Proceedings of the Southern Conference on Programmable Logic (SPL), pp. 133-137, March 2010.

[14] C. Ruething, A. Agne, M.Happe, C. Plessl, "Exploration of ring oscillator design space for temperature measurements on FPGAs," in Proceedings of the International Conference on Field Programmable Logic and Applications (FPL), pp. 559-562, August 2012.

[15] N. Rahmanikia, A. Amiri, H. Noori, F. Mehdipour, "Performance evaluation metrics for ring-oscillator-based temperature sensors on FPGAs: A quality factor," Integration, the VLSI Journal, vol 57, pp. 81-100, March 2017.

[16] G. Payá-Vayá, C. Bartels, H. Blume, "Small footprint synthesizable temperature sensor for FPGA devices," Journal of Systems Architecture, vol 76, pp. 28-38, May 2017.

[17] C. Lefebvre, J.L. Montero, L. Rubio, "Implementation of a fast relative digital temperature sensor to achieve thermal protection in Zynq SoC technology," Microelectronics Reliability, to be published, 2017.

[18] Altera Inc., Altera temperature sensor IP core user guide, UG-01074, 2015.

[19] J. Li, Sh. Feng, Y. Zhang, Ch. Wang, X. He, "Optimized thermal sensor allocation for field-programmable gate array temperature measurements based on self-heating test," Microelectronics Journal, vol 60, pp. 55-59, February 2017.

[20] A. Agne, H. Hangmann, M. Happe, M. Platzner, C. Plessl, "Seven recipes for setting your FPGA on fire - a cookbook on heat generators," Microprocessors and Microsystems, vol 38, pp. 911-919, November 2014.

[21] P. Chen, M.C. Shie, Z.Y. Zheng, Z.F. Zheng, C.Y. Chu, "A fully digital time-domain smart temperature sensor realized with 140 FPGA logic elements," IEEE Transactions on Circuits and Systems I: Regular Papers, vol 54, pp. 2661-2668, December 2007. 